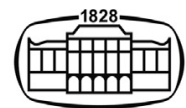

AKADÉMIAI KIADÓ

IMAGING

\title{
Did we turn a blind eye? The answer is simply there. Peripheral pulmonary vascular thrombosis in COVID-19 patients explains sudden worsening of clinical conditions
}

\author{
PIERLEONE LUCATELLI* ๑, MAURIZIO DEL MONTE, \\ GIANLUCA DE RUBEIS, GIULIA CUNDARI, \\ MARCO FRANCONE, VALERIA PANEBIANCO and \\ CARLO CATALANO
}

\section{SHORT}

COMMUNICATION

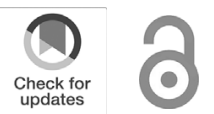

IMAGING 12 (2020) 1, 4-7

DOI: $10.1556 / 1647.2020 .00002$

(c) 2020 The Author(s)

*Corresponding author. Department of Radiological, Oncological and Pathological Sciences, Sapienza University of Rome, V.le Regina Elena 324, 00161, Rome, Italy. Tel.:+3906 4455602; Fax: +3906 490243

E-mail: pierleone.lucatell@gmail.com
Department of Radiological, Oncological and Pathological Sciences, "Sapienza" University of Rome, Rome, Italy

Received: May 21, 2020 • Accepted: May 26, 2020

\section{ABSTRACT}

COVID-19 patients suffering sudden worsening of clinical conditions have an atypical peripheral pulmonary arterial obstruction at computed tomography pulmonary angiogram (CTPA), poorly associated to deep venous thrombosis (DVT), suspicious for thrombotic in situ nature rather than embolic.

\section{KEYWORDS}

COVID-19, pulmonary vascular thrombosis, computed tomography pulmonary angiography, CTPA

\section{Introduction}

Several efforts have been made to understand why some COVID-19 patients experience a sudden worsening of clinical conditions [1]. Just on May 7th, McGonagle et al. [2] provided an insight on the possible mechanism of action of COVID-19 infection, suggesting how extensive diffuse alveolar and pulmonary interstitial inflammation may trigger extensive pulmonary immunothrombosis.

Despite an emerging evidence on the presence of pulmonary embolism (PE) in COVID-19 patients [3-5] has been reported, this mechanism has not been unmasked.

Interestingly all these series reported a high incidence of peripheral localization of the obstruction (44-100\% [3-5]), less observed in PE not associated to COVID-19 [6, 7]. Moreover Poissy et al. [4] reported an increased prevalence of PE in intensive care unit (ICU) COVID-19 patients compared with control period (20.6 vs. $6.1 \%)$ with low rate of associated deep venous thrombosis (DVT) (respectively of 13.6 vs. $58.3 \%$ ).

We hypothesized that vascular obstruction could be pulmonary in situ rather than being only of embolic nature. This hypothesis is also supported by the reported pro-coagulative status in COVID-19 patients $[8,9]$. The objective of our study was to localize pulmonary obstruction in association with COVID-19 infection using pulmonary computed tomography pulmonary angiogram (CTPA).

\section{Materials and methods}

This retrospective study was approved by our institutional review board. It followed the ethical guidelines of the Declaration of Helsinki. Written informed consent was waived. Two 
authors had access to the study data. No author has any conflict of interest to declare in relation to this study.

\section{Patients}

From March 3rd to April 8th 2020 a total of 275 patients were positively tested for COVID-19 with nasopharyngeal swab samples followed by RT-PCR assay to confirm the diagnosis. Of these, 28/275 (10.1\%) (23/28 [82.1\%] male, mean age $67.7 \pm 15.2$ years old), with worsening conditions (dyspnea; mean $\mathrm{PaO}_{2} / \mathrm{FiO}_{2}$ ratio $261,45 \pm 106$; mean DDimer 2,168 $\pm 1,596 \mathrm{ng} / \mathrm{mL}$ ) clinical suspicions of PE, and negative evidence of peripheral thrombosis at Doppler Ultrasound performed a CTPA.

\section{CT Pulmonary Angiography (CTPA)}

Technique. CTPA angiograms were acquired on a dual source computed tomography (CT) scanner (Somatom Definition, Siemens, Erlangen, Germany) after i.v. injection of 50-70 mL of iodinated contrast agent (Iomeprol $400 \mathrm{Mg}$ $\mathrm{I} / \mathrm{mL}$, Bracco Imaging, Milan, IT) at a flow rate of $4 \mathrm{~mL} / \mathrm{s}$, with the use of a bolus-tracking technique and a threshold of $180 \mathrm{HU}$ in the main pulmonary trunk.

Imaging Analysis. CTPA were independently analyzed by two blinded radiologists (PL, MDM with 12 and 9 years of experience). In cases of discordance, a simultaneous reading to reach consensus was achieved. Categorization of vascular obstruction level was performed according to Mastora et al. score [10]. This score permits distinction of the contribution to total obstruction burden into central score (mediastinal arteries), peripheral score (lobar and segmental arteries) and global CT score. Additionally, presence/absence of indirect CTPA signs of pulmonary hypertension and overload of the right heart (inferior vena cava reflux, interventricular septum flatting/blowing, interventricular septum flatting/ blowing; superior vena cava flatting/blowing; right/left ventricles ratio; pulmonary artery/ascending aorta ratio) were assessed [11].

\section{Results}

Of the 275 patients admitted to our hospital for COVID-19, $28 / 275(10.1 \%)(23 / 28$ [82.1\%] male, mean age $67.7 \pm 15.2$ years old) performed a CTPA for suspected PE. All patients $(28 / 28$ [100\%]) presented CT findings of arterial thrombosis with involvement of mediastinal (2/28 [7.1\%]), lobar (5/28 [17.9\%]) and segmental arteries (28/28 [100\%]). The median global CT score index was 10.0 (CI 95\% 7.4-12.0, range 2.048.0 ), the median central score (mediastinal arteries) was 0.0 (CI 95\% 0.0-0.0, range 0.0-8.0) and the median peripheral score (lobar and segmental arteries) was 10.0 (CI 95\% 7.412.0, range 2.0-48.0). Twenty-one of the 28 (75.0\%) patients had indirect CTPA signs of pulmonary hypertension and overload of the right heart (Fig. 1). In particular, superior vena cava flatting/blowing was found in $14 / 28$ (50.0\%) patients, inferior vena cava reflux in $7 / 28$ (25.0\%) and interventricular septum flatting/blowing in 9/28 (32.1\%). A 4chambers right/left ventricles ratio $>1$ was found in $16 / 28$ (57.1\%) patients (median 1.1 [CI 95\% 1.0-1.2], range 1.0$1.5)$ and pulmonary artery/ascending aorta ratio $>1$ in $6 / 28$ (21.4\%) (median 1.1 [CI 95\% 1.0-1.1], range 1.0-1.1). The incidence of associated DVT was 2/28 (7.1\%). Moreover, one patient underwent invasive mechanical thrombectomy with reported systolic pulmonary pressure of $55 \mathrm{mmHg}$; his pulmonary angiography depicted a peripheral perfusion defect with subsegmental associated thrombosis (Fig. 2).

\section{Discussion}

Our results underline the high prevalence of peripheral pulmonary artery obstruction with concomitant signs of pulmonary hypertension in COVID-19 patients. Moreover, pulmonary obstruction was poorly associated with DVT, perfectly matching the recently proposed underlying pathogenesis as "pulmonary in situ" rather than embolic.

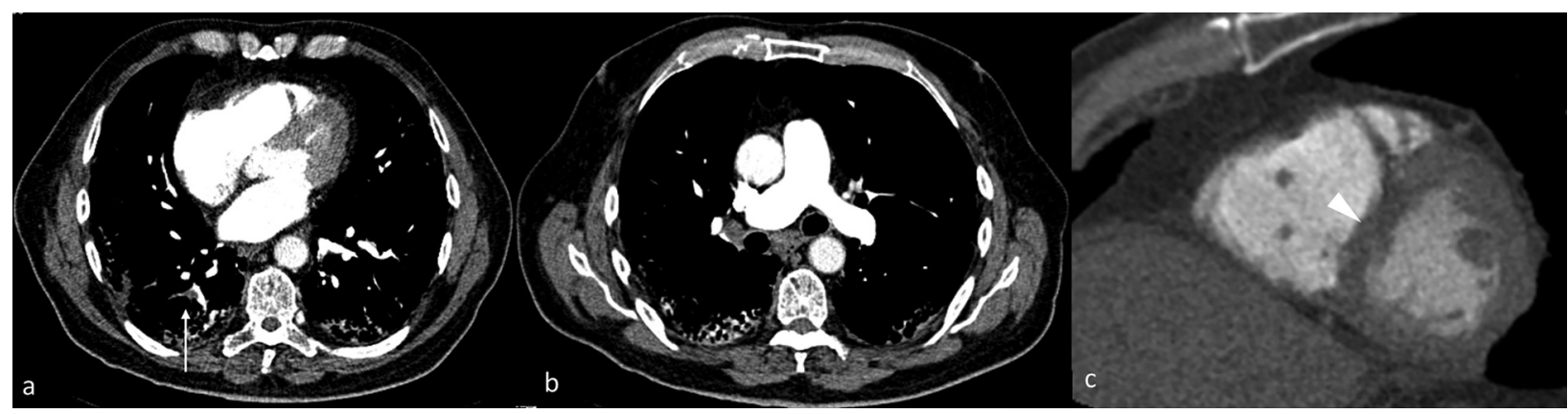

Figure 1. Clinical case of 70-year-old male. On axial plane CT pulmonary angiogram in pulmonary arterial phase (a), it is possible to observe a segmental peripheral branch occlusion (score 4) for the right inferior lobe (arrow); Figure $b$ showed, in the same phase of Figure $a$, the absence of obstruction in main branch of pulmonary arteries. In Figure $c$, a 2-chambers view middle plane of the heart, the interventricular septum is flattening as an indirect sign of pulmonary hypertension (arrowhead) 


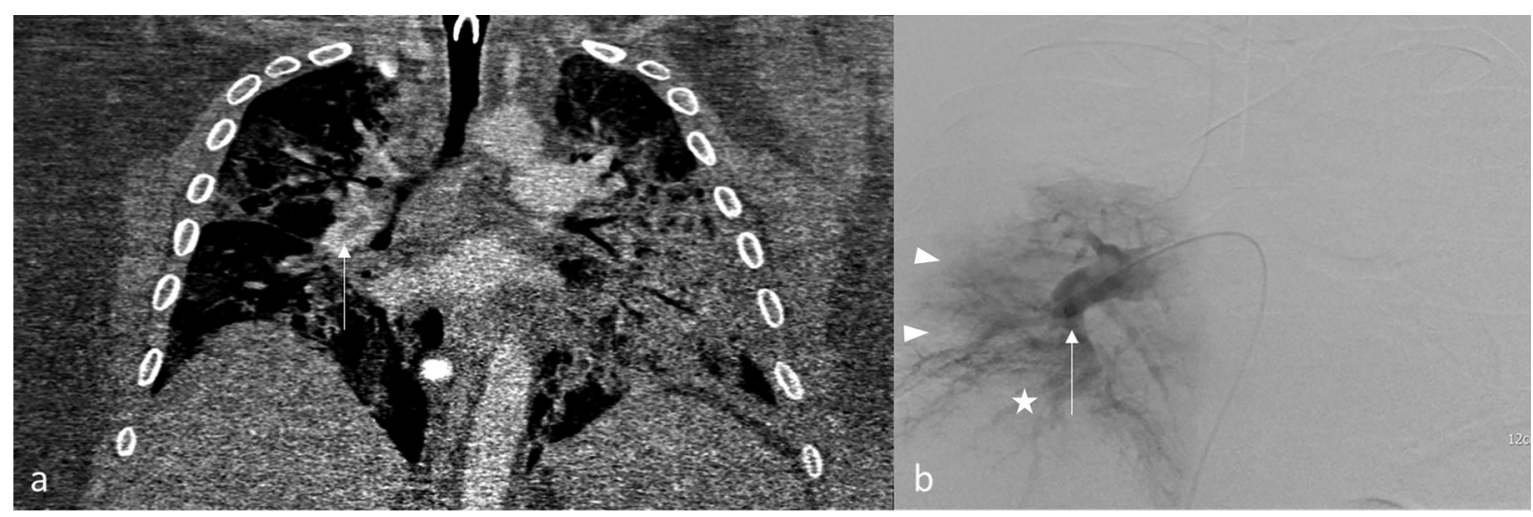

Figure 2. Clinical case of 38-year-old male with $\mathrm{BMI}=38$. Coronal plane CT angiogram in pulmonary arterial phase (a) showed an hypodense clot in the lobar branch of the right pulmonary arteries (arrow). The obstruction was confirmed by digital subtracted angiography (b) (arrow) associated with micro thrombosis of a peripheral sub-segmental vessel (star) and triangular shaped perfusion defect (arrowhead)

Considering our results (atypical pulmonary obstruction poorly associated to DVT) and those reported in other series (1-3), it seems rationale to believe, rather than the embolic nature, in a different underlying pathogenesis of pulmonary arterial thrombosis, being the associated DVT relatively low. Moreover the topographic distribution of the obstructed vessels was atypical (mostly peripheral); in fact in typical DVT-PE isolated peripheral vascular involvement is rare $[6,7]$.

Therefore, the presence of a peripheral in situ pulmonary vascular thrombosis with pre-capillary pulmonary hypertension, explains the persistent dyspnea, as theorized by McGonagle et al. [2]. These findings may explain the high mortality of COVID-19 patients in ICU despite proper mechanical ventilation. In fact, these patients could have both a ventilation deficit (non-aerated lung) and perfusion defect (pulmonary in situ vascular thrombosis) leading to an extreme alteration of ventilation/perfusion ratio and overload of the right heart. Thus, the onset of pulmonary in situ vascular thrombosis could explain the rapid worsening of the clinical conditions with hypoxemia and hypocapnia.

This concept is even supported by the reported evidence of decreased areas of peripheral perfusion corresponding to peripheral lung opacities, demonstrated at dual energy CT examinations [12], that perfectly matches with the findings of subpleural lack of perfusion depicted at the pulmonary angiography in course of mechanical thrombectomy of our patient (Fig. 2).

Limitations of the study were its retrospective nature, the limited sample size and the single energy acquisition.

In conclusion, considering pulmonary vascular thrombosis as the trigger event for worsening clinical condition, CTPA should be a part of COVID-19 patient's radiological flow-chart, being an "all-in-one" technique for vascular occlusion assessment and right heart involvement evaluation.

Authors' contribution: All authors listed made a significant contribution to the manuscript with regards to its conception, writing and final approval. All authors read and approved the submission of the manuscript. The manuscript has not been published and is not being considered for publication elsewhere, in whole or in part in any language.

Conflict of interest: None.

\section{REFERENCES}

[1] Saba L, Sverzellati N: Is COVID evolution due to occurrence of pulmonary vascular thrombosis? J Thorac Imaging 2020. https:// doi.org/10.1097/RTI.0000000000000530. 32349055.

[2] McGonagle D, O’Donnell JS, Sharif K, Emery P, Bridgewood C: Immune mechanisms of pulmonary intravascular coagulopathy in COVID-19 pneumonia. Lancet Rheumatol 2020. https://doi.org/ 10.1016/S2665-9913(20)30121-1.

[3] Grillet F, Behr J, Calame P, Aubry S, Delabrousse E: Acute pulmonary embolism associated with COVID-19 pneumonia detected by pulmonary CT angiography. Radiology 2020: 201544. https://doi.org/10.1148/radiol.2020201544.

[4] Poissy J, Goutay J, Caplan M, Parmentier E, Duburcq T, Lassalle F: Pulmonary embolism in COVID-19 patients: awareness of an increased prevalence. Circulation 2020. https://doi.org/10.1161/ CIRCULATIONAHA.120.047430.

[5] Leonard-Lorant I, Delabranche X, Severac F, Helms J, Pauzet C, Collange O, Moliere S: Acute pulmonary embolism in COVID-19 patients on CT angiography and relationship to D-dimer levels. Radiology 2020: 201561. https://doi.org/10.1148/radiol.2020201561.

[6] Diffin DC, Leyendecker JR, Johnson SP, Zucker RJ, Grebe PJ: Effect of anatomic distribution of pulmonary emboli on interobserver agreement in the interpretation of pulmonary angiography. AJR Am J Roentgenol 1998; 171: 1085-9.

[7] de Monye W, van Strijen MJ, Huisman MV, Kieft GJ, Pattynama PM: Suspected pulmonary embolism: prevalence and anatomic distribution in 487 consecutive patients. Advances in New Technologies Evaluating the Localisation of Pulmonary Embolism (ANTELOPE) Group. Radiology 2000; 215: 184-8. 
[8] Amgalan A, Othman M: Exploring possible mechanisms for COVID-19 induced thrombocytopenia: unanswered questions. J Thromb Haemost JTH 2020; 18(6): 1514-6. https://doi.org/10. 1111/jth.14832.

[9] Chen T, Wu D, Chen H, Yan W, Yang D, Chen G: Clinical characteristics of 113 deceased patients with coronavirus disease 2019: retrospective study. BMJ 2020; 368: m1091.

[10] Mastora I, Remy-Jardin M, Masson P, Galland E, Delannoy V, Bauchart JJ: Severity of acute pulmonary embolism: evaluation of a new spiral CT angiographic score in correlation with echocardiographic data. Eur Radiol 2003; 13: 29-35.

[11] Moore AJE, Wachsmann J, Chamarthy MR, Panjikaran L, Tanabe Y, Rajiah P: Imaging of acute pulmonary embolism: an update. Cardiovasc Diagn Ther 2018; 8: 225-43.

[12] Lang M, Som A, Mendoza DP, Flores EJ, Reid N, Carey D, : Hypoxaemia related to COVID-19: vascular and perfusion abnormalities on dual-energy CT. Lancet Infect Dis 2020; S14733099(20): 30367-4. https://doi.org/10.1016/S1473-3099(20)30367-4. 